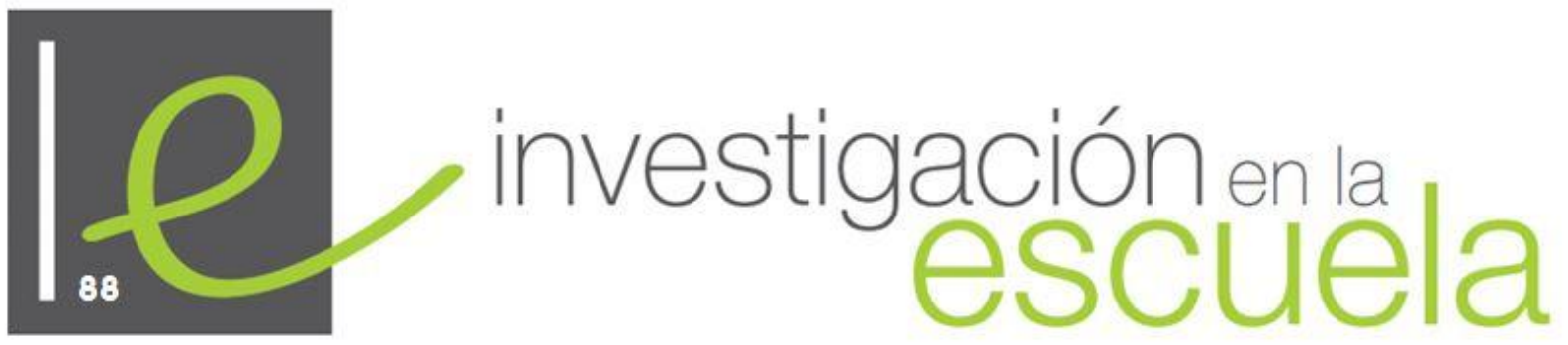

Revista académica evaluada por pares y de acceso abierto

\title{
"Drug Research": una secuencia contextualizada de indagación sobre mitosis, cáncer y creación del conocimiento científico ${ }^{1}$.
}

\author{
Jordi Domènech Casal \\ Universidad Autónoma de Barcelona \\ España
}

Citación: Domènech, J. (2016). "Drug Research": una secuencia contextualizada de indagación sobre mitosis, cáncer y creación del conocimiento científico. Investigación en la escuela, 88, 93-111. Recuperado de www.investigacionenlaescuela.es/articulos/R88/R88-6

Resumen: la adquisición de habilidades y perspectivas científicas es clave en la educación científica de los futuros ciudadanos. A pesar de los esfuerzos que se realizan en este sentido, el alumnado conserva una visión estereotipada y rígida del método y el conocimiento científico. Se propone una actividad de indagación sobre la mitosis y el cáncer en la que el alumnado vive en primera persona el proceso investigador, de-construyendo el método científico. En la actividad, los alumnos parten de biopsias ficticias para iniciar una investigación en la que publican artículos científicos, redactan proyectos y realizan experimentos, en el marco de dinámicas de construcción del conocimiento

\footnotetext{
${ }^{1}$ Reflexiones sobre la contextualización y la auto-regulación de los aprendizajes contenidas en este artículo se enmarcan en el trabajo del grupo LIEC (Llenguatge i Ensenyament de les Ciències), grupo de investigación consolidado de la Universitat Autònoma de Barcelona (referencia 2009SGR1543) por AGAUR (Agència d'Ajuts Universitaris i de Recerca) y financiado por la Dirección General de Investigación, Ministerio de Educación y Ciencia (referencia EDU-201238022-C02-02).
} 
científico. Los resultados de la aplicación y sus efectos en las concepciones del alumnado se discuten junto con una encuesta realizada al alumnado.

Palabras clave: "indagación”; "mitosis"; "cáncer"; "comunicación científica”

\section{Drug Research: a contextualized inquiry activity on mitosis, cancer, and creation of} scientific knowledge.

Abstract: acquiring scientific skills and perspectives is a key point in science education. In spite of the efforts on this field, students keep a rigid and stereotyped image of the creation of the scientific knowledge. We propose an inquiry didactic sequence where students participate as investigators in a project relating mitosis and cancer, de-constructing the scientific method. In the activity, students analyse fake biopsies to undertake a research in which they publish scientific articles, write projects and perform experiments in the frame of social dynamics of construction of scientific knowledge. A survey and the results of its application are discussed regarding the effects on students' conceptions about scientific knowledge.

Key words: "inquiry"; "mitosis"; “cáncer"; "scientific communication”.

Drug Research: une séquence contextualisée d'apprentissage par enquête sur la mitose, le cancer et la création du savoir scientifique.

Résumé: L'acquisition d'habiletés et perspectives scientifiques est un point clé chez l'éducation scientifique. Malgré les efforts mis en œuvre, les élèves maintiennent une image rigide et stéréotypée de la méthode et le savoir scientifique. On propose une séquence d'enseignement sur la mitose et le cancer qui se déroule au long d'une enqûete où l'élève participe en première personne dans la création du savoir scientifique et la déconstruction de la méthode scientifique. L'activité démarre de l'analyse de biopsies simulées, à partir de laquelle, les élèves publient des articles scientifiques, écrivent un projet scientifique et font des expériences, dans le cadre de dynamiques sociales de création du savoir scientifique. Les résultats de l'application et ses effets sur les conceptions des élèves sont discutés à l'aide d'un sondage.

Mosts clé: "enquête"; "mitose"; "cáncer"; "communication scientifique".

\section{Introducción}

La mitosis y la regulación del ciclo celular son conceptos clave de la biología celular que forman parte del currículum de la Educación Secundaria Obligatoria de $3^{\circ}$ y $4^{\circ}$ de ESO. Varios autores afirman que el alumnado de secundaria suele presentar dificultades en la comprensión de la mitosis (Smith y Kindfield, 1999; Riemeier y Gropengieber, 2008; Dikmenli, 2012) y su estudio suele realizarse de un modo excesivamente memorístico. Eso dificulta su transferencia por parte del alumnado a contextos relevantes, como el cáncer o las células madre.

Así mismo, en referencia a los aspectos de investigación científica, a menudo el alumnado conserva una imagen mitológica y excesivamente deshumanizada de la ciencia y los científicos (Campanario, 1999), desprovista de los componentes económicos y sociales que influyen en la creación del conocimiento científico. Con el doble objetivo de profundizar en la comprensión de la mitosis y su transferencia a los procesos tumorales y acercar al alumnado a la naturaleza de la investigación científica, nos planteamos el desarrollo de una actividad en la que el alumnado llevara a cabo una investigación en primera persona sobre el cáncer y la mitosis. 


\section{Marco teórico}

Más allá del dominio de los principales modelos científicos (el ciclo del agua, el modelo atómico, la tectónica de placas, la evolución de las especies,...), la formación de ciudadanos competentes científicamente implica también la adquisición de las habilidades y estrategias que conducen a la construcción del conocimiento científico (elaboración de hipótesis, diseño de experimentos, análisis de datos, comunicación de resultados...) y el desarrollo de una actitud crítica para posicionarse científicamente ante hechos o dilemas socio-científicos. (Hodson, 1994; Pedrinacci et al, 2012)

Las iniciativas pedagógicas afrontan este reto de dos modos: por un lado enseñar la ciencia como indagación (a menudo en puntos concretos del currículum, como la materia de Ciencias para el Mundo Contemporáneo, o unidades didácticas específicas sobre el método científico) y, por el otro, enseñar la ciencia mediante la indagación (Enseñanza de las Ciencias Basada en la Indagación, en adelante, ECBI) (Hodson, 1994; Barberá y Valdés, 1996; Llewellyn, 2005). Todo ello en base a estrategias distribuidas a lo largo del currículum, que ponen el acento en formular actividades investigadoras y la instrumentalización de las habilidades de razonamiento científico por parte del alumnado para la construcción del conocimiento científico (Osborne y Dillon, 2008; Caamaño, 2002 y 2012).

Varios autores defienden que la ECBI, además de fomentar el interés y apropiación de los modelos científicos por parte del alumnado (Rocard et al, 2007) constituye una vía para conseguir aprendizajes realmente transferibles a nuevos contextos (Hodson, 1994; Osborne y Dillon, 2008). Se ha propuesto para las actividades ECBI un itinerario estandarizado lineal con varias etapas, en un intento de reproducir el proceso científico de creación del conocimiento en la escuela (Bogner, Boudalis y Sotiriou, 2012): 1) Formular preguntas investigables 2) Dar prioridad a las evidencias 3) Analizar las evidencias 4) Formular una explicación basada en las evidencias 5) Conectar la explicación con el conocimiento científico 6) Comunicar y justificar la explicación 7) Reflexionar sobre el proceso y el aprendizaje.

Trabajos recientes ponen en evidencia la importancia de enfocar las actividades investigadoras hacia la comunicación y de qué modo la relación cognitivo-lingüística entre pensarescribir-hablar -potenciada mediante el uso de andamios didácticos de carácter lingüístico- puede ayudar a desarrollar habilidades de razonamiento científico (Sanmartí, 2008). Aun así, la comprensión de la naturaleza de la ciencia no sólo necesita el desarrollo de habilidades científicas (diseñar experimentos, sacar conclusiones de datos) sino también la comprensión y reflexión sobre las dinámicas sociales de construcción de conocimiento en comunidad (García-Carmona, 2012; Garritz, 2006).

El proyecto C3 (Domènech, 2013a) sobre la Creación del Conocimiento Científico tiene por objetivo el desarrollo y aplicación de actividades didácticas orientadas a que el alumnado viva en primera persona la creación del conocimiento científico para obtener una percepción personal de la naturaleza del conocimiento científico como un proceso (no un producto) de construcción social (no de descubrimiento). Como estrategias clave, las actividades del proyecto usan la enseñanza de las ciencias mediante la indagación (ECBI), el uso de andamios lingüísticos para promover el desarrollo de habilidades científicas, y el trabajo en contextos que emulen y estimulen procesos de creación del conocimiento científico. El proyecto pretende superar la visión rígida, cartesiana e idealizada del método científico, evidenciando la parte "democrática", "serendípica", humanizada y constructivista que varios autores reclaman (Fernández et al, 2002). 


\section{Descripción de la experiencia}

La actividad se desarrolló y aplicó con un total de 90 alumnos de $4^{\circ}$ de ESO de la materia optativa de Biología y Geología durante tres cursos académicos en dos institutos de secundaria distintos: el 2012-2013 en el instituto Marta Mata, de Montornès del Vallès, y los cursos 2013-2014 y 2014-2015 en el instituto de Vilanova del Vallès. En el primero se aplicó sólo una parte de las actividades, de forma prospectiva, que fueron mejoradas y aplicadas en los dos cursos siguientes en su versión más completa que se describe en este artículo.

La actividad parte de datos ficticios de pacientes en tratamiento con varios medicamentos anti-tumorales experimentales. A lo largo de varias sesiones (7-9) con varios formatos comunicativos (artículo científico, conversación informal, redacción de proyectos,...), estrategias didácticas (lectura en grupo, trabajo práctico con microscopios, análisis de datos,...) y agrupaciones de alumnos (individual, por parejas, en grupos,...), el alumnado investiga, en base a los resultados de las biopsias, la eficacia de los distintos medicamentos. La actividad se compone de 6 etapas:

- Etapa 1: Análisis de biopsias.

- Etapa 2: Diseño de experimentos.

- Etapa 3: Comunicación científica, escribir un artículo científico.

- Etapa 4: Redacción de proyectos de investigación.

- Etapa 5: Preparación y observación de muestras.

- Etapa 6: Reflexión sobre la experiencia.

A lo largo de la actividad, se recogieron observaciones en el aula y producciones del alumnado como evidencias del proceso, además de recoger en una encuesta las impresiones del alumnado sobre los cambios en sus concepciones sobre el rol/figura del investigador científico y el conocimiento científico. Los materiales de la actividad están disponibles para su descarga libre en una página web en el formato de Webquest, apta para ser propuesta directamente a los alumnos .

\section{Etapa 1: Análisis de biopsias. Extrayendo conclusiones de datos en diferentes formatos.}

La experiencia parte de 20 fichas médicas ficticias elaboradas a propósito para la actividad, que se distribuyen al azar entre el alumnado (una ficha por alumno) ${ }^{2}$. Las fichas contienen los resultados (ficticios) de biopsias pre-test y post-test practicadas a ratones con los que se han testado varios medicamentos antitumorales candidatos (Zanton L, Heferedoxina,...) a diferentes dosis (10 U, $30 \mathrm{U}$ ) y duración del tratamiento (1 mes/3 meses), correspondiéndose cada ficha a una sola combinación experimental. Después de presentar al alumnado el proceso de la mitosis y su relación con el cáncer, se pide al alumnado que, de forma individual, calcule el índice mitótico de las dos imágenes en su ficha (biopsias antes y después del tratamiento) para determinar si el tratamiento es eficaz o no. La ficha proporciona andamios didácticos para calcular el índice mitótico y construir un histograma con los resultados. Por razones del diseño de la actividad, las células que se observan son de origen vegetal (ese detalle no se comunica al alumnado). La oportunidad de esa elección se comenta más adelante.

Al terminar esta etapa, cada alumno habrá discriminado las diferentes fases de la mitosis, y habrá representado en un histograma los índices mitóticos obtenidos.

\footnotetext{
${ }^{2}$ Cada ficha especifica el medicamento, la dosis y duración del tratamiento e imágenes de biopsias antes y después del tratamiento
} 


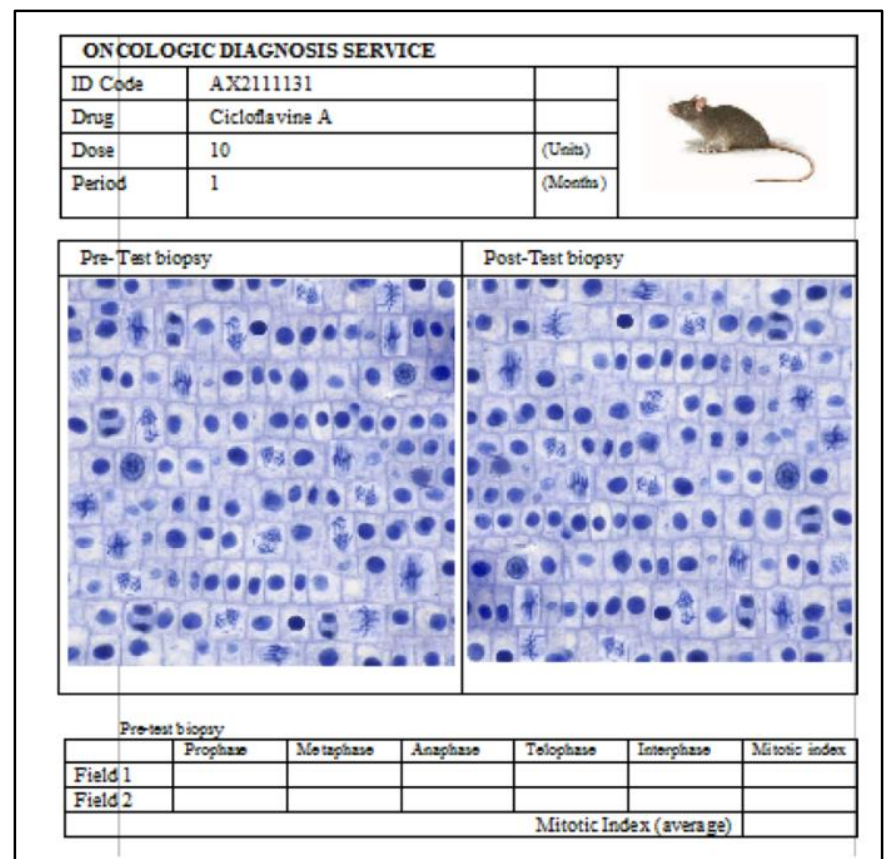

Imagen 1. Imagen de una de las fichas de análisis que se proporcionan a los alumnos.

En esa primera etapa, como materiales de apoyo, se proporcionan al alumnado documentos y vídeos sobre el proceso de la mitosis y su relación con el cáncer. Para entrenar al alumnado en el análisis de ese tipo de datos, se han desarrollado y aplicado actividades de apoyo, opcionales en la secuencia: por un lado una ficha de entrenamiento, o Training Session, y por el otro, una actividad plástica sobre la meiosis y la mitosis. Estas actividades no se describen aquí por su carácter opcional en la secuencia, pero pueden hallarse en la web de la actividad .

El profesor acompaña al alumnado mostrando una ficha de ejemplo y modelando el proceso de atribución "Ésta célula parece que tiene el núcleo algo raro...debe ser una de las fases de la mitosis... ¿Se distinguen los cromosomas? Sí...parecen alineados...entonces, ¿Qué sería? ¿Una metafase?".

Los alumnos manifiestan desconcierto cuando en el proceso tienen dudas sobre alguna de las células y se les pide qué decidirían en ese caso. Se aprovecha para discutir con ellos el hecho que en los análisis existen márgenes de error y que es necesario determinar qué errores son asumibles y cuáles no (es asumible diagnosticar cáncer a una persona que no lo tiene, recomendando un segundo análisis, pero no lo es diagnosticar como sano a alguien enfermo).

\section{Etapa 2. Diseño de experimentos. El equipo de investigación. Identificando variables, detectando tendencias.}

En esta segunda etapa se pide a los alumnos que intercambien, en diálogo informal (deambulación libre por el aula) la información de su ficha de análisis, con el objetivo de buscar una ficha que complemente la información que se halla en la suya. Esto implica un subliminal análisis de variables: los alumnos descubren por sí mismos que no pueden sacar conclusiones si más de una variable (medicamento, dosis, tiempo) es diferente. 


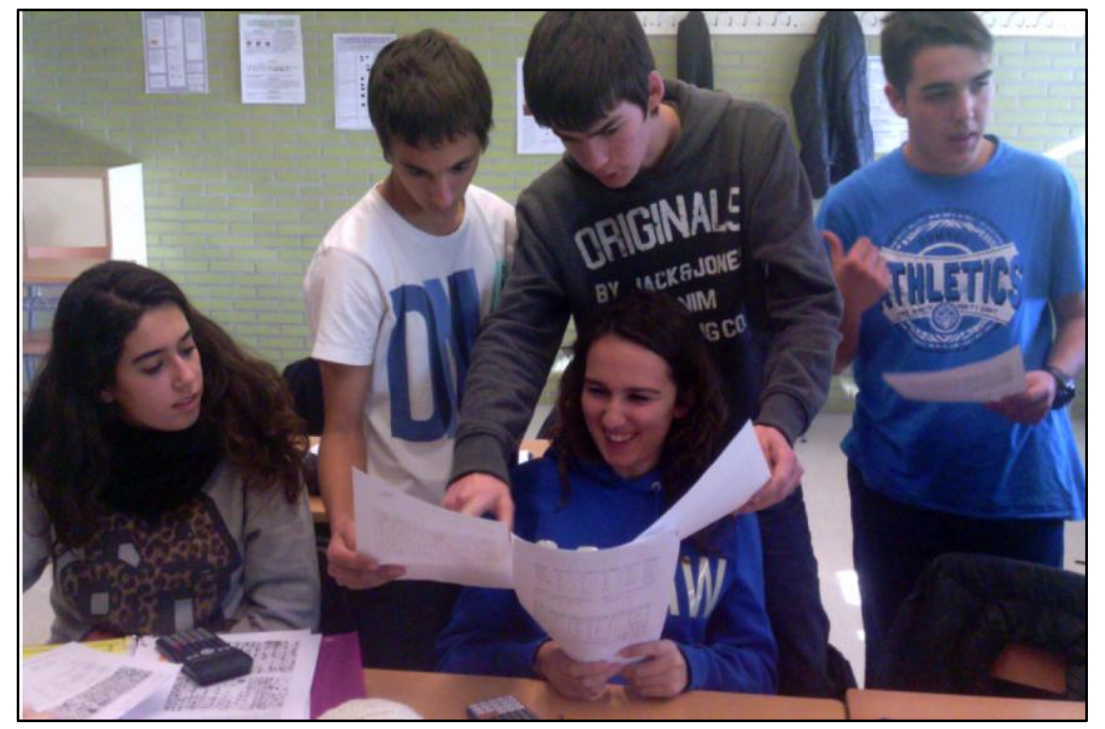

Imagen 2. Un equipo de investigación

Se pide a los alumnos que formen parejas cuyas fichas constituyan un experimento del que puedan sacarse conclusiones. En esta fase empiezan a observarse dinámicas colaborativascompetitivas. Incluso, en la formación de parejas de tratamientos, de forma excepcional algunos alumnos han quedado sin pareja, al no encontrar otro tratamiento compatible con el suyo que no tuviera pareja. Esto se ha resuelto creando en algún caso, equipos de tres alumnos. Para ayudar al alumnado a valorar la comparación de los resultados de las distintas fichas, el profesor circula por el aula proponiendo preguntas "Qué cosas cambian entre un tratamiento y otro? ¿Podemos atribuir el cambio a alguna de las variables? ¿Qué ha pasado al cambiar la dosis? ¿Por qué crees que ha pasado? ¿Significa eso algo con respecto al medicamento?’”.

\section{Etapa 3. Comunicación científica. Consejos editoriales.}

Una vez formadas las parejas, cada pareja debe redactar un artículo científico en el que exponga los resultados y conclusiones de su experimento, usando obligatoriamente el vocabulario específico relacionado con la mitosis y el cáncer. Como andamio didáctico de apoyo para la elaboración del artículo, se proporciona a los alumnos una plantilla con iniciadores de frase y conectores gramaticales que permitan identificar la funcionalidad argumentativa y estilo lingüístico de las distintas secciones de un artículo científico, además de un ejemplo de artículo científico escolar sobre otra temática -más sencillo- para ayudar a identificar la estructura del artículo científico.

Se hace especial énfasis a los alumnos para que en las conclusiones establezcan qué otros tratamientos serían necesarios para llegar a conclusiones más seguras o concluyentes. Antes de iniciar la escritura del artículo, se comenta en gran grupo qué tipo de informaciones deberían incluirse en cada uno de los apartados. Se presenta a los alumnos una lista de revistas científicas ficticias (Tabla 1), cada una de ellas con diferentes estándares de calidad escolares y se comunica al alumnado que como culminación de la actividad, podrán elegir qué tratamientos quieren observar al microscopio para completar su investigación, pero que, para hacerlo, deberán elaborar un proyecto, en el que será de gran importancia su currículum como científicos, en especial, la revista en la que consigan publicar su artículo. Cada pareja elige a qué revista envía su artículo, consciente de que de ello depende su éxito en las próximas etapas. 
Tabla 1

Listado de revistas

\begin{tabular}{|c|c|c|c|c|}
\hline & $\begin{array}{c}\text { Archives } \\
\text { of Cell } \\
\text { Biology }\end{array}$ & $\begin{array}{c}\text { Journal of } \\
\text { Cell } \\
\text { Research }\end{array}$ & $\begin{array}{l}\text { Trends in } \\
\text { Cancer } \\
\text { Research }\end{array}$ & $\begin{array}{l}\text { Cancer } \\
\text { Research } \\
\text { Journal }\end{array}$ \\
\hline $\begin{array}{l}\text { Los tratamientos que se comparan no } \\
\text { permiten llegar a conclusiones. } \\
\text { Gráficos y pies de figura correctos, } \\
\text { redacción correcta, aunque poco clara. } \\
\text { No hay conclusiones erróneas, aunque } \\
\text { pueden estar incompletas o poco } \\
\text { relacionadas con los conceptos básicos de } \\
\text { mitosis y cáncer. }\end{array}$ & $\sqrt{ }$ & $\sqrt{ }$ & $\sqrt{ }$ & $\sqrt{ }$ \\
\hline $\begin{array}{l}\text { Se ha hecho una buena elección de los } \\
\text { datos a comparar y el objetivo de la } \\
\text { comparación. } \\
\text { Redacción clara y bien argumentada. } \\
\text { Puede haber conclusiones incompletas, } \\
\text { pero están bien relacionadas con los } \\
\text { conceptos básicos de mitosis y cáncer. }\end{array}$ & & $\sqrt{ }$ & $\sqrt{ }$ & $\sqrt{ }$ \\
\hline $\begin{array}{l}\text { Se anticipa qué otros tratamientos serían } \\
\text { necesarios para llegar a más conclusiones. } \\
\text { El artículo saca todas las conclusiones } \\
\text { correctas posibles. La argumentación es } \\
\text { consistente y bien relacionada con } \\
\text { conceptos básicos. }\end{array}$ & & & $\sqrt{ }$ & $\sqrt{ }$ \\
\hline $\begin{array}{l}\text { Además de las condiciones anteriores, los } \\
\text { resultados se defienden de un modo que } \\
\text { (sin perder el rigor) se ve su aplicabilidad, } \\
\text { se conectan con el conocimiento } \\
\text { científico y posibles investigaciones } \\
\text { futuras. }\end{array}$ & & & & $\sqrt{ }$ \\
\hline
\end{tabular}

Nota: la lista de revistas es en el fondo una plantilla de evaluación de los artículos de modo que los consejos editoriales constituyen en el fondo una actividad de co-evaluación. Cada revista tiene un distinto nivel de exigencia: mientras Archives of Cell Biology sólo pide uno de los requisitos de la columna de la izquierda, Trends in Cancer Research pide tres.

Hecho esto, se constituyen los Consejos Editoriales ${ }^{3}$. Los alumnos se reparten entre los cuatro consejos de las cuatro revistas y evalúan para su publicación en la revista los artículos que han sido enviados. Si un artículo es rechazado, es necesario argumentar de forma oral por qué y según el caso se permite a los autores enviarlo a otra revista o mejorarlo para cumplir los requisitos.

\footnotetext{
${ }^{3} \mathrm{La}$ formación de los Consejos Editoriales es una oportunidad para que el alumnado analice las producciones de otros compañeros, algo interesante, pues en ocasiones el alumnado aprende más de ejemplos alternativos que de complejos andamios didácticos abstractos.
} 
Esta fase es interesante, pues aparecen preferencias, amiguismos o rivalidades y sus efectos en la selección de artículos. No se dan a los alumnos instrucciones de incompatibilidad (como no poder evaluar su propio artículo), pues éstas son propuestas y adoptadas de forma natural por el alumnado. El curso 2014-2015 los alumnos llegaron incluso a publicar ediciones digitales de las revistas (ejemplos disponibles en: http://bit.ly/1HMjn67, http://bit.ly/1aLsnLt).

$\mathrm{Al}$ final de esta fase se discuten con los alumnos las relaciones de poder e influencia o los criterios de selección de publicaciones y sus efectos en la visualización y promoción de determinadas vías de investigación, en contraste con el modelo del "investigador libre" comprometido sólo con el conocimiento. Se han producido discusiones entre algunos alumnos respecto a la calidad de los artículos o la imparcialidad de los jueces, que han sido retomadas en un debate breve, con las siguientes claves para el debate:

- ¿Hasta qué punto ha sido independiente vuestro juicio? ¿Qué medidas se podrían adoptar para que lo fuera más?

- ¿Qué es más importante: la calidad de los datos, las conclusiones que se saca de ellos, o la forma en que se comunican?

- ¿Qué factores en el mundo real pueden hacer que un consejo editorial sea más o menos independiente y fiable?

\section{Etapa 4. Redactando proyectos de investigación, consiguiendo financiación. Diseño de experimentos.}

En esta etapa, cada equipo de investigación (pareja) debe buscar otros dos equipos y constituir un grupo de investigación de 6 alumnos para escribir juntos un proyecto ${ }^{4}$ en el que solicitar tratamientos con muestras reales que observar en el laboratorio. Esto conlleva que los objetivos que cada equipo ha configurado en base a sus resultados queden re-contextualizados ante los datos proporcionados por otros equipos. Esta redefinición de los objetivos según el contexto (poco presente en los modelos escolares de metodología científica) es algo muy habitual en la investigación científica.

Aunque parecida a la dinámica anterior de creación de los equipos de investigación, la creación de los grupos incorpora un aspecto significativo: a cada grupo se le concederán más o menos tratamientos en función no sólo de la calidad del proyecto, sino también de la calidad de los currículums investigadores de los participantes (revista en la que se han aceptado los artículos). Esto hace que al intentar diseñar proyectos, los alumnos tengan en cuenta los dos aspectos: por un lado, la unión de los datos de los distintos artículos debe formar un corpus temático lo suficientemente compacto (mismo medicamento, dosis o tiempo) para poder diseñar nuevos experimentos a partir de él. Por otro lado, tienen en cuenta también la calidad de los artículos de los equipos con quienes deciden colaborar. De hecho, se ha observado que algún equipo ha desarrollado una estrategia "compensatoria", al decidir colaborar con un equipo con buena publicación y otro con el que se configura un buen proyecto.

\footnotetext{
${ }^{4} \mathrm{Al}$ construir el proyecto, los alumnos deben usar el "paisaje" de conocimiento actual a partir de sus artículos, identificando los "vacíos" para establecer objetivos.
} 
Tabla 2

Guía para la presentación del proyecto.

\begin{tabular}{|c|l|}
\hline DESCRIPTORES & \multicolumn{1}{|c|}{ EXPLICACIÓN } \\
\hline $\begin{array}{c}\text { Descripción breve del } \\
\text { proyecto }\end{array}$ & $\begin{array}{l}\text { Antecedentes y objetivo del proyecto. Indicar qué tratamientos se } \\
\text { solicitan. Justificar, para cada tratamiento, con qué objetivo concreto } \\
\text { se solicita, cuáles son los posibles resultados, y qué indicaría. }\end{array}$ \\
\hline $\begin{array}{c}\text { Anteriores aportaciones } \\
\text { de los miembros del } \\
\text { grupo en el campo de la } \\
\text { investigación biomédica }\end{array}$ & $\begin{array}{l}\text { Explicar vuestras líneas de investigación anteriores y en qué } \\
\text { consistían, de modo que se evidencie la relevancia de las } \\
\text { investigaciones anteriores en el proyecto que se presenta. }\end{array}$ \\
\hline $\begin{array}{c}\text { Publicaciones recientes } \\
\text { de los miembros del } \\
\text { equipo de investigación }\end{array}$ & $\begin{array}{l}\text { Formato: } \\
\text { Pérez, C., Auró, R. Las arañas se vuelven sordas cuando les quitan } \\
\text { todas las patas (2011). Journal of Cancer Research, 22, 11-13 }\end{array}$ \\
\hline
\end{tabular}

Nota: para redactar el proyecto, se proporciona a los alumnos un andamio, una versión simplificada de los formularios para las solicitudes de ayudas a la financiación. Los proyectos son presentados por vía telemática en entorno virtual al profesor, que actúa como institución evaluadora.

Anticipar "qué indicaría si" implica haber desarrollado un modelo de lo que sucede y examinar cómo se manifestaría en los distintos tratamientos. Esta estrategia de anticipar las conclusiones de cada resultado ya había sido usada anteriormente con éxito (Domènech, 2013b y en evaluación). La inclusión en la redacción del proyecto por parte de los alumnos de otras actividades anteriores en las que hayan desarrollado habilidades científicas constituye una oportunidad como reflexión/portfolio de habilidades científicas. Para ayudar a los miembros de cada proyecto, el profesor circula entre los grupos y les invita a identificar qué "agujeros" de conocimiento dejan los artículos de sus componentes, o qué tendencias descubren: “¿Existe algún efecto general de dosis? ¿Uno de los medicamentos parece especialmente potente, pero no se sabe si funciona igual a dosis bajas?".

\section{Etapa 5. Preparación y observación de muestras. Dando prioridad a la observación, testando hipótesis.}

A partir de los proyectos presentados, y en función de la calidad de cada proyecto, el profesor concede a cada grupo una proporción distinta de los tratamientos solicitados. Algunos grupos con buenos proyectos que habían solicitado seis tratamientos han obtenido cinco, mientras que otros, habiendo solicitado tres han obtenido sólo uno.

Las muestras "reales" que el profesor proporciona a los alumnos son en realidad secciones milimétricas de raíz de cebolla. En los tratamientos donde deberían observarse muchas mitosis se han seleccionado secciones del ápice de raíces de cebolla puestas en contacto con agua durante dos días, mientras que en el resto se han usado raíces no expuestas al agua (por tanto, con menos mitosis).

Los alumnos obtienen las muestras pre-preparadas con Orceína $\mathrm{A}^{5}$, e identificadas por las condiciones que representan (medicamento, dosis, tiempo). Ellos completan la preparación de la muestra con Orceína B y observan las muestras al microscopio -usando protocolos convencionales

\footnotetext{
${ }^{5}$ En las muestras observadas, el pre-tratamiento de las muestras con Orceína A (de color rojo vivo) colabora a mantener el equívoco de encontrarse ante muestras reales de biopsias de ratón.
} 
para la preparación de las muestras y la observación al microscopio- para obtener la información para completar su proyecto.

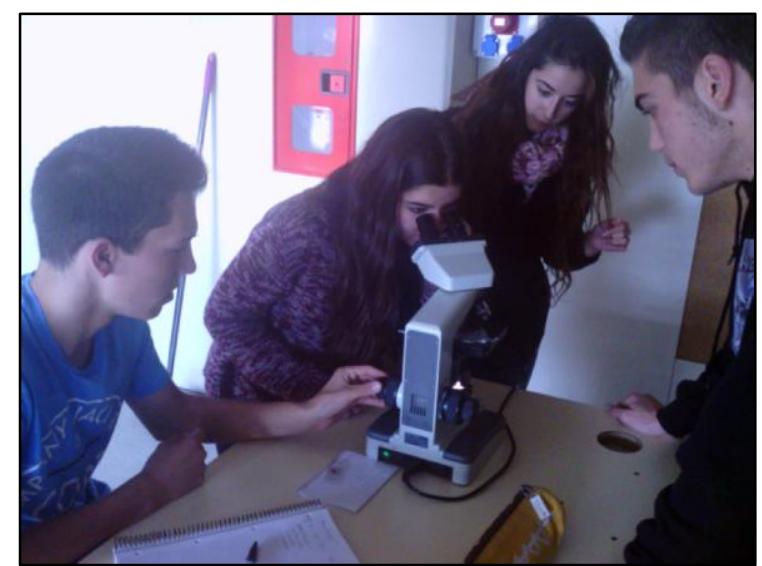

Imagen 3. Análisis de muestras.

En los cursos en los que se ha desarrollado la experiencia ha habido siempre una parte de las muestras que no se han podido observar correctamente, en ocasiones debido a dificultades en el procedimiento de tinción, que han conllevado discusión sobre los objetivos de cada uno de los pasos del protocolo de tinción-observación. Lejos de suponer un impedimento en la actividad, conviene incluir este aspecto como una parte más de la ciencia real: los experimentos no siempre salen como prevemos, y conviene saber sacar el máximo de información, incluso de los experimentos fallidos.

Llegados a este punto, el ciclo se cierra de nuevo. Al final de la sesión de laboratorio, cada grupo presenta de forma oral informal sus conclusiones y se inicia un diálogo en gran grupo. El profesor aprovecha este momento para elaborar una recapitulación del proceso seguido hasta el momento para que los alumnos se den cuenta de volvemos a encontrarnos en el punto "inicial": extraer conclusiones de datos.

La actividad investigadora termina en esta etapa, y aunque no en ella no se solicita a los alumnos la elaboración de ningún producto, es posible continuar la secuencia elaborando un póster científico sobre los resultados del proyecto (lo que supondría una nueva reformulación de objetivos, dada la pérdida de algunas muestras). Este tipo de actividad se desarrolla como parte del proyecto C3 en otras partes del currículum de la materia (Domènech, 2014).

\section{Etapa 6. Reflexionando sobre el proceso. Ética y epistemología de la investigación científica.}

Como último paso, y a continuación de la recapitulación de la actividad, se desarrolla un último debate breve sobre los aspectos éticos y epistemológicos de la experiencia, en particular sobre el modelo publicación/financiación para la investigación científica, y los aspectos bioéticos. Las claves para el debate son las siguientes:

¿Hasta qué punto son fiables y exactos los datos y conclusiones del análisis inicial? ¿Qué puede hacerse para mejorar eso?

- ¿Qué idea ha tenido prioridad para ti: la curación de los pacientes o el éxito como científico/a?

- ¿Qué problema supone el sistema de selección de proyectos y publicación de artículos? ¿Cómo lo resolverías?

- ¿En algún momento has considerado si la cantidad de ratones que su usaban y si sufrían o no 
en el proceso? ¿Cómo se contrapone eso con el desarrollo de medicamentos?

- ¿Es correcto usar en humanos medicamentos experimentados sólo en ratones? ¿Con qué humanos los testarías? ¿Qué problemas supone eso?

\section{Resultados}

La actividad propuesta representa los distintos pasos estándar de la ECBI (Bogner, Boudalis y Sotiriou, 2012), pero propone un itinerario en el que éstos transcurren de forma paralela o desordenada respecto a las propuestas tradicionales bajo el nombre de "método científico": se inicia de forma prospectiva, los objetivos se reformulan en el curso de la investigación y se obtienen conclusiones sobre cuestiones que no se habían planteado. La componente de juego de rol de la actividad ha generado un gran interés entre el alumnado, algo ya descrito por otros autores como implicación pedagógica de las actividades en las que se pide al alumno un rol indagador, como la ECBI (Rocard et al, 2007): los alumnos se han mostrado muy motivados, y, en determinadas partes de la secuencia didáctica (consejos editoriales, escritura de proyectos) incluso competitivos. Consideramos que el desarrollo de un rol investigador en el marco de una propuesta de aprendizaje activo ha sido de utilidad para movilizar las habilidades del alumnado.

Para evaluar el cambio en las concepciones respecto a la creación del conocimiento científico se solicitó a 28 alumnos participantes en la experiencia el curso 2013-2014 que rellenaran una encuesta comparando sus concepciones antes y después de la actividad. En ella, se les solicitaba que eligieran de una lista cerrada las palabras que mejor describían distintos sintagmas. Los resultados (Figura 1) muestran que los alumnos consideran que la actividad ha cambiado sus concepciones sobre la investigación científica y los científicos.

Destaca el cambio relativo al rango de certeza del conocimiento científico y el cambio en las aptitudes para ser buen científico/a. Es preocupante el poco éxito del término "objetivo" como descripción del conocimiento científico, aún después de la actividad. Ya observamos en actividades anteriores (Domènech, 2014) situaciones en la misma línea o incluso peores, en las que en actividades de creación social de conocimiento los alumnos incrementaban su apoyo al término "subjetivo" para describir el conocimiento científico, lo que pone en evidencia un conflicto entre las visiones constructivista y socio-construccionista de la ciencia. 


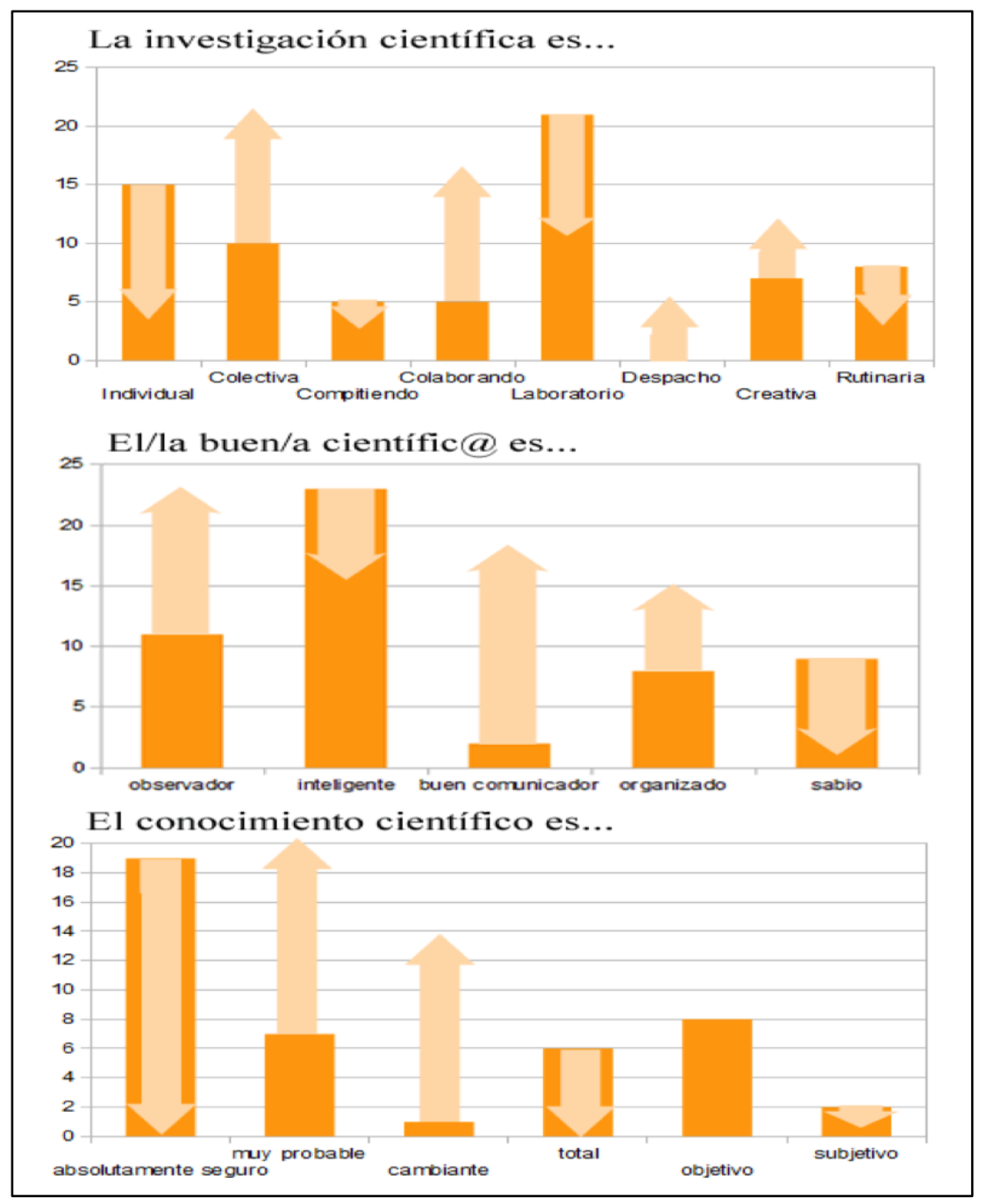

Figura 1. Concepciones previas (columnas) y cambios producidos (flechas)

Respecto a la comprensión de los modelos de mitosis y cáncer, los resultados obtenidos en el examen (en el que se ha pedido al alumnado que representara las biopsias que corresponderían a la intervención de determinados medicamentos) muestran que el alumnado ha mejorado de forma notable respecto a grupos de cursos anteriores la comprensión del proceso de mitosis, especialmente en la identificación de sus fases y su relación con el ciclo celular y el cáncer. No obstante se observan carencias en el dominio del vocabulario de algunos términos específicos (replicación, centrosoma, huso mitótico,...) algo observado por otros autores en experiencias prácticas de enseñanza de la división celular (Riemeier y Gropengieber, 2008). Los defectos en la sistematización de los conocimientos en actividades ECBI han sido descritos anteriormente por otros autores (Viennot, 2011), proponiéndose alternar las actividades puramente investigativas con sesiones o "talleres" en los que se estructure de forma más sistematizada los conocimientos, una alternativa que estamos valorando junto con el uso de portfolios.

En los proyectos presentados por los alumnos ${ }^{6}$ se aprecia el uso frecuente y adecuado de conectores gramaticales, como puede observarse en el siguiente extracto: "estudios anteriores verifican que el medicamento Zanton $L$ ralentiza su efectividad sin una mejora notable de la

${ }^{6}$ Otros ejemplos de proyectos presentados por el alumnado están disponibles en: http://bit.ly/1aLxLOC 
enfermedad. Además, en las biopsias extraídas se detecta un alto índice de toxicidad. Por otro lado, el medicamento Heferodoxina consigue reducir notablemente el índice mitótico del individuo aunque la mejora no es excesiva. En cambio, al aplicar Heferodoxina y Zanton L a la misma vez, obtenemos una reducción drástica de la enfermedad en el individuo".

El uso de andamios didácticos lingüísticos como herramienta para mejorar el razonamiento científico del alumnado para la elaboración de comunicaciones científicas, ya testado con anterioridad en el proyecto C3 (Domènech, 2013a, 2014 y 2015), ha resultado una herramienta útil. En la evaluación de las producciones del alumnado (artículos, proyectos) hemos detectado respecto a experiencias anteriores una mejora substancial de los textos del alumnado, especialmente en lo que refiere a la distinción entre resultados y conclusiones, la argumentación y la correlación correcta de datos como objetivos, resultados y conclusiones. Aunque es difícil determinarlo con exactitud (diferentes edades de los alumnos, diferente instituto), consideramos que la presentación de ejemplos contextualizados o andamios que reproduzcan la silueta del texto a obtener han ayudado a mejorar los resultados. Para posteriores experiencias se considera una vía interesante el trabajo previo con actividades de lectura de artículos científicos reales que actúen como modelos.

\section{Conclusiones}

Consideramos que la "deconstrucción" de las propuestas lineales identificadas en la práctica escolar como "método científico" (Figura 2) es necesaria para dar al alumnado una visión más cercana a la ciencia real, en el que diferentes fases se interrelacionan entre sí en un proceso en espiral que no tiene, realmente, ningún inicio ni final definidos, y puede recorrer itinerarios diversos sujetos a la creatividad y no por ello incorrectos científicamente (Figura 3) ${ }^{7}$.
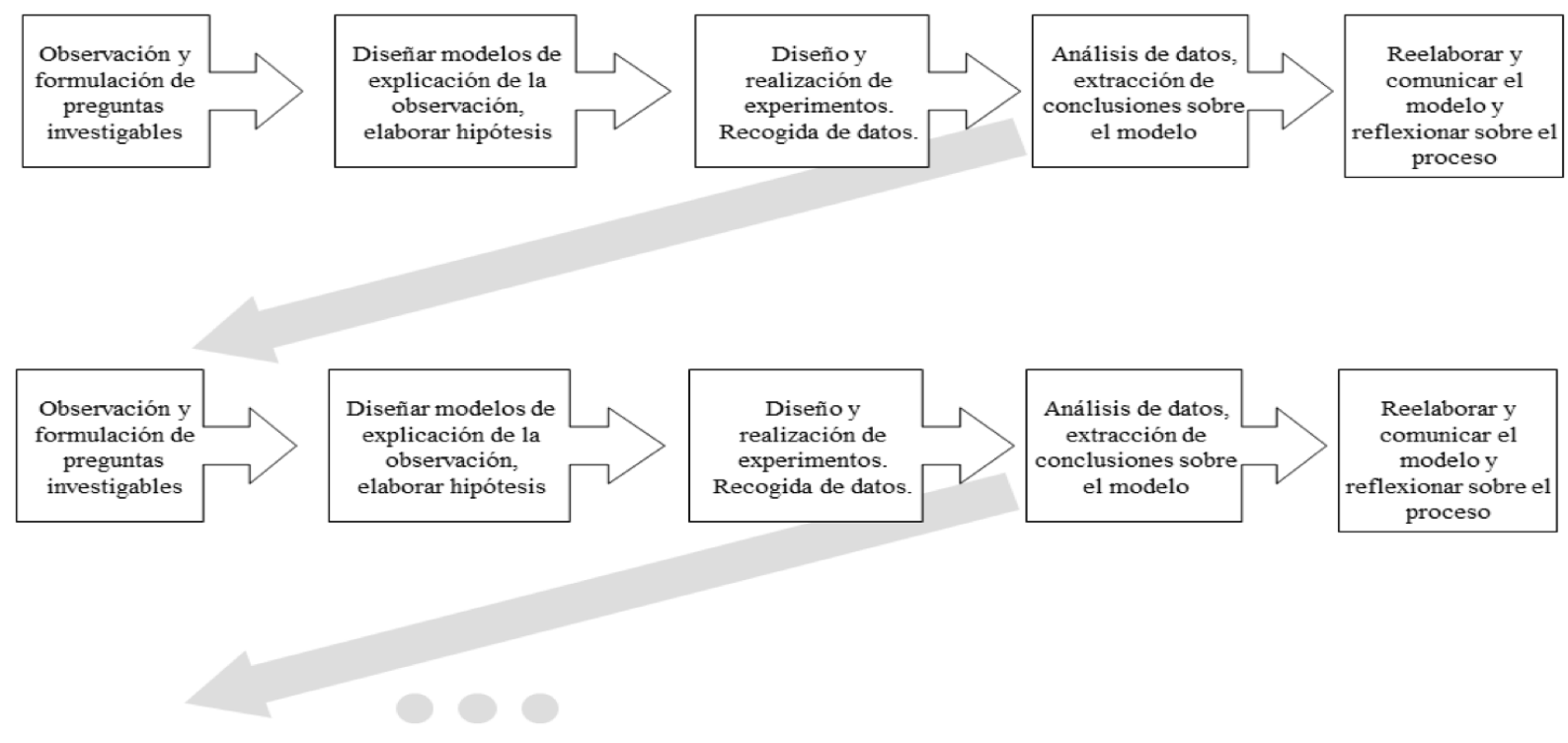

Figura 2. Modelo secuencial.

\footnotetext{
${ }^{7}$ Las secuencias didácticas tradicionales de trabajo según el método científico transcurren linealmente (Figura1), muchas veces forzando al participante a procesos o pasos innecesarios, que en la ciencia real son realizados de un modo más fluido y multidireccional, y que puede dar lugar a distintos procesos creativos como muestra la Figura 2.
} 


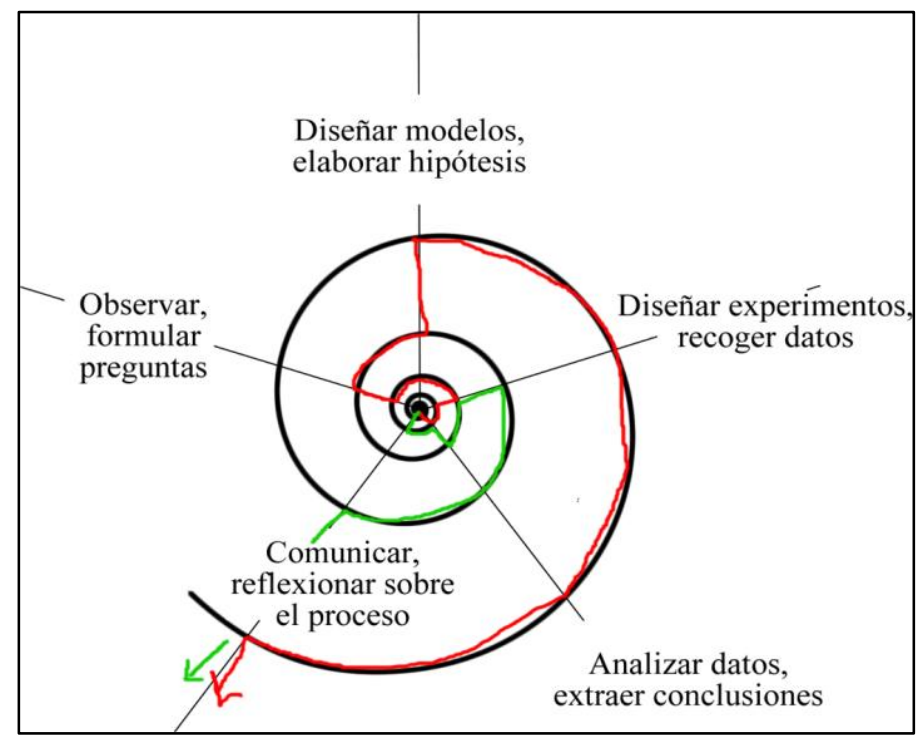

Figura 3. Modelo integrado

La actividad combina las perspectivas de la ECBI y las WebQuest y cumple varios aspectos que consideramos clave y desde el proyecto C3 proponemos como claves para el desarrollo de actividades de aprendizaje significativo en ciencias:

- Recorre los distintos procesos de creación del conocimiento científico, promoviendo la habilidad del alumnado de hacerse preguntas, diseñar experimentos, analizar datos y sacar conclusiones, para hacer que los contenidos se adquieran mediante su instrumentalización (Pedrinacci et al, 2012).

- Usa estrategias didácticas variadas (lectura en grupo, trabajo práctico con microscopios, análisis de documentos, debate...) y distintos espacios y artefactos (aula, comunicación virtual, laboratorio) diversificando los canales de acceso al conocimiento.

- Promueve formatos comunicativos científicos diversos (artículo científico, conversación informal, redacción de proyectos, debate en gran grupo...) hacia los que se proyecta la creación social de conocimiento.

- Se desarrolla en múltiples organizaciones sociales del aula (individual, por parejas, en grupos,...) en las que el alumno es gestor y promotor del conocimiento (Pujolàs, 2009; Egues y Aldanondo, 2012).

- Genera o presenta un contexto que da sentido a la actividad (Sanmartí, Burgoa y Nuño, 2011) y conecta la ciencia con sus implicaciones sociales y tecnológicas en la realidad cercana del alumnado.

- Ofrece retos intelectuales y andamios didácticos de distintos niveles (rúbricas, ejemplos estándar, producciones de compañeros,...) para promover el aprendizaje y la autonomía creciente del alumnado.

Sin ser un aspecto de importancia en lo relativo a lo pedagógico, el idioma inglés y formato WebQuest de la actividad permite que ésta pueda ser usada por profesorado de otros centros docentes. De hecho, a lo largo de la actividad se han usado tres idiomas: el inglés (en los materiales de las actividades) el catalán (en la mayor parte de las producciones de los alumnos) y el castellano (para la redacción del proyecto), lo que para los alumnos no ha supuesto dificultades.

Como vías de desarrollo de la actividad, consideramos que deberían considerarse los 
siguientes aspectos:

- La conexión con los aspectos éticos, tecnológicos y de aplicación médica es esencial, aunque debe gestionarse con delicadeza, detectando si hay alumnos que pudieran ser especialmente sensibles a la temática por casos familiares. Debe en todo caso, dejarse muy claro al alumnado que las técnicas de análisis y criterios usados no son ajustados para la interpretación de datos de pacientes humanos reales, y que la actividad constituye un modelo simplista del diagnóstico de la enfermedad.

- El uso de imágenes de mitosis vegetales y tejidos vegetales es una respuesta a la limitación técnica de conseguir buenas imágenes o tejidos disponibles de origen animal. Conviene estar atentos a las concepciones erróneas que eso puede suscitar (forma de las células, citocinesis...), aunque éste es un punto de difícil solución: mantener hasta el final de la actividad el equívoco de que analizamos muestras reales es importante para la implicación del alumnado y se aconseja ser vagos al dar explicaciones respecto al origen de las muestras al alumnado.

- Asociar un efecto concreto a nivel molecular (interferencia con los microtúbulos, bloqueo del cinetocoro,...) a cada medicamento candidato permitiría asociar los estados de "parada" de la mitosis a distintos medicamentos, poniendo de relevancia los pasos y mecanismos moleculares del proceso (no sólo la posición y movimiento de los cromosomas).

- Aunque no se han observado en la aplicación, la actividad puede promover concepciones erróneas que deben ser objeto de atención, como por ejemplo, la visión de la mitosis como un proceso intrínsecamente perjudicial, o desvinculado de procesos clave, como el desarrollo o incluso la herencia, como han descrito otros autores (Lewis y Wood-Robinson, 2000). Esta experiencia se inscribe en el proyecto C3 sobre la creación del conocimiento científico, junto con otras experiencias relacionadas (Domènech, 2014 y 2015). La actividad ha sido catalogada en la Aplicació de Recobriment del Currículum (ARC) del Departament d'Ensenyament de la Generalitat de Catalunya, la red ENCIENDE y el directorio WebQuest.cat de la Comunitat Catalana de WebQuest. El autor agradece a los alumnos participantes su participación en la evaluación de la secuencia didáctica.

\section{Referencias}

Barberá, O. \& Valdés, P. (1996) El trabajo práctico en la enseñanza de las ciencias: una revisión. Enseñanza de las Ciencias, 14(3), 365-379.

Bogner, F., Boudalis, A.K. \& Sotiriou, S. (Eds.) Pathway. Best Practices of Inquiry-Based Science Education. Methods and Activities. Pallini Attikis, Greece: Epinoia.

Caamaño, A. (2002). ¿Cómo transformar los trabajos prácticos tradicionales en trabajos prácticos investigativos? Aula de Innovación Educativa, 113, 21-26.

Caamaño, A. (2012). ¿Cómo introducir la indagación en el aula? Los trabajos prácticos investigativos. Alambique: didáctica de las ciencias experimentales, 70, 83-91.

Campanario, J.M. (1999). La ciencia que no enseñamos. Enseñanza de las Ciencias, 17(3), 397-410.

Dikmenli, M. (2010). Misconceptions of cell division held by student-teachers in biology: a drawing analysis. Scientific Research and Essay, 5, 235-247.

Dodge, B. (1995). WebQuests: A Technique for Internet-Based Learning. The Distance Educator, 1(2), 10-13. 
Domènech, J. (2013a). Secuencias de apertura experimental y escritura de artículos en el laboratorio: un itinerario de mejora de los trabajos prácticos en el laboratorio. Enseñanza de las Ciencias, 31 (3) 249-262.

Domènech, J. (2013b). Escritura de artículos y diseño de experimentos: andamios para escribir, pensar y actuar en el laboratorio. Enseñanza de las Ciencias, número especial Congreso ENSE Ciencias , 1085-1089.

Domènech, J. (2014). Una secuencia didáctica en contexto sobre evolución, taxonomía y estratigrafía basada en la indagación y la comunicación científica. Alambique, Didáctica de las Ciencias Experimentales, 78, 51-59.

Domènech, J. (2015). Una secuencia didáctica de modelización, indagación y creación del conocimiento científico en torno a la deriva continental y la tectónica de placas. Revista Eureka sobre Enseñanza y Divulgación de las Ciencias, 12(1), 186-197.

Domènech, J. (en evaluación). Gene Hunting: una secuencia contextualizada de indagación alrededor de la expresión génica, la investigación in silico y la ética en la comunicación biomédica. Revista Eureka sobre Enseñanza y Divulgación de las Ciencias..

Egues, J. y Aldanondo, A. (2012). Aprendizaje cooperativo: un valor añadido. Cuadernos de pedagogía, 428, 34-36.

España, E., Rueda, J.A. \& Blanco, A. (2013) Juegos de rol sobre el calentamiento global. Actividades de enseñanza realizadas por estudiantes de ciencias del Máster en Profesorado de Secundaria. Revista Eureka sobre Enseñanza y Divulgación de las Ciencias, 10, 763-779.

Fernández, I., GIL, D., Carrascosa, J., Cachapuz, A. y Praia, J. (2002). Visiones deformadas de la ciencia transmitidas por la enseñanza. Enseñanza de las Ciencias, 20(3), 477-488.

García-Carmona, A. (2012). Cómo enseñar naturaleza de la ciencia (NDC) a través de experiencias escolares de investigación científica. Alambique, Didáctica de las Ciencias Experimentales, 72, 55-63.

Garritz, A. (2006). Naturaleza de la ciencia e indagación: cuestiones fundamentales para la educación científica del ciudadano. Revista Iberoamericana de Educación, 42, 127-152.

Riemeier, T. y Gropengießer, H. (2008). On the roots of difficulties in learning about cell division process-based analysis of students' conceptual development in teaching experiments. International Journal of Science Education, 30(7), 923-939.

Hodson, D. (1994). Hacia un enfoque más crítico del trabajo de laboratorio. Enseñanza de las Ciencias, 12(3), 299-313.

Lewis, J. y Wood-Robinson, C. (2000). Genes, chromosomes, cell division and inheritance - do students see any relationship? International Journal of Science Education, 22(2), 177-195.

Llewellyn, D. (2005). Teaching High School Science through Inquiry: A case study approach. California: Corwin Press \& NSTA press.

Osborne, J. \& Dillon, J. (2008). Science Education in Europe: Critical Reflections. Londres: Nuffield Foundation.

Pedrinacci, E., Caamaño, A., Cañal, P. y De Pro, A. (2012). 11 ideas clave. El desarrollo de la competencia cientifica. Barcelona: Graó.

Pujolàs, P. (2009). 9 ideas clave. El aprendizaje cooperativo. Barcelona, Graó.

Rocard, M., Csermely, P., Jorde, D., Lenzen, D., Walberg-Heriksson, H. \& Hemmo, V. (2007). Science Education Now: a new pedagogy for the future of Europe. Report for the European Comission. Recuperado de: «http://ec.europa.eu/research/sciencesociety/document_library/pdf_06/report-rocard-on-science-education_en.pdf »

Sanmartí N. (2008) Escribir para aprender ciencias. Aula de Innovación Educativa, 175, 29-32.

Sanmartí, N., Burgoa, B. \& NUÑO, T. (2011) ¿Por qué el alumnado tiene dificultad para utilizar sus conocimientos científicos escolares en situaciones cotidianas? Alambique, Didáctica de las Ciencias Experimentales, 67, 62-69. 
Smith, M. \& Kindfield, A. (1999). Teaching Cell Division. The American Biology Teacher, 61(5), 366371.

Viennot, L. (2011). Els molts reptes d'un ensenyament de les Ciències basat en la indagació: ens aportarà múltiples beneficis en l'aprenentatge? Ciències, 18, 22-36.

\section{Sobre el Autor}

Autor: Jordi Domènech-Casal

Institución: Institut de Granollers (Barcelona); Grupo LIEC, Departamento de Didáctica de las Matemáticas y las Ciencias Experimentales, Universidad Autónoma de Barcelona.

E-mail: jdomen44@xtec.cat

Información biográfica: Jordi Domènech-Casal (1976) es Doctor en Biología y Licenciado en Humanidades. Entre los años 1999-2007 se dedicó a la investigación básica en genética molecular y química inorgánica (Universidad de Barcelona, Université París VII, Universitá di Bologna) y a la divulgación científica. Es Profesor de Secundaria desde 2008 (actualmente en el Institut de Granollers, Barcelona) y desde 2013 asesor de formación de profesorado en el Departament d'Ensenyament de la Generalitat de Cataluña. Coordina el grupo de trabajo de profesorado de ciencias EduWikiLab, el proyecto C3 de enseñanza competencial de las ciencias y el proyecto IndComp de evaluación interdisciplinar por competencias. Es miembro del grupo Llengua $i$ Ensenyament de les Ciències LIEC (Universitat Autònoma de Barcelona), profesor asociado en la misma Universidad y miembro del consejo asesor de la revista Cuadernos de Pedagogía. Ha publicado en varias revistas de ámbito nacional e internacional artículos alrededor de la auto-regulación de los aprendizajes, la Enseñanza de las Ciencias Basada en la Indagación y el Aprendizaje Basado en Proyectos. Web personal:

https://jordidomenechportfolio.wordpress.com/

ORCID: orcid.org/0000-0002-7324-0000 


\section{Investigación en la Escuela}

Revista académica evaluada por pares y de acceso abierto

Número 88

1 de octubre de 2016

ISSN 2443-9991

\section{()}

SOMERIIGHISRESEEVED Los/as lectores/as pueden copiar, mostrar, y distribuir este artículo, siempre y cuando se de crédito y atribución al autor/es y a Investigación en la Escuela, se distribuya con propósitos no-comerciales, no se altere o transforme el trabajo original. Más detalles de la licencia de Creative Commons se encuentran en http://creativecommons.org/licenses/by-nc-sa/3.0 Cualquier otro uso debe ser aprobado en conjunto por el autor/es, o Investigación en la Escuela.

Contribuya con comentarios y sugerencias en la web de la revista. Por errores y sugerencias contacte a investigacionescuela@,ddcc.uhu.es 


\title{
Investigación en la escuela
}

Consejo de dirección: Ana Rivero García (Universidad de Sevilla), Nicolás de Alba Fernández (Universidad de Sevilla), Pedro Cañal de León (Universidad de Sevilla), Francisco F. García Pérez (Universidad de Sevilla), Gabriel Travé González, (Universidad de Huelva), Francisco F. Pozuelos Estrada (Universidad de Huelva)

\author{
Dirección: Ana Rivero García y Nicolás de Alba Fernández \\ Técnico de edición: Francisco Javier López Sánchez
}

\section{Consejo editorial}

José Félix Angulo Rasco. Universidad de Cádiz Rosa Ma Ávila Ruiz. Universidad de Sevilla Pilar Azcárate Goded. Universidad de Cádiz Juan Bautista Martínez Rodríguez. Universidad de Granada

Nieves Blanco García. Universidad de Málaga Fernando Barragán Medero. Universidad de La Laguna José Carrillo Yáñez. Universidad de Huelva José Contreras Domingo. Universidad de Barcelona. Luis C. Contreras González. Universidad de Huelva Ana $\mathbf{M}^{\mathbf{a}}$ Criado García-Legaz. Universidad de Sevilla Rosario Cubero Pérez. Universidad de Sevilla José $\mathbf{M}^{\mathbf{a}}$ Cuenca López. Universidad de Huelva Jesús Estepa Giménez. Universidad de Huelva Rafael Feito Alonso. Universidad Complutense (Madrid)

Francisco José García Gallardo. Universidad de Huelva

Soledad García Gómez. Universidad de Sevilla J. Eduardo García Díaz. Universidad de Sevilla
Fernando Hernández Hernández. Universidad de Barcelona

Salvador Llinares Ciscar. Universidad de Alicante Alfonso Luque Lozano. Universidad de Sevilla Rosa Martín del Pozo. Universidad Complutense (Madrid)

José Martín Toscano. IES Fernando Herrera (Sevilla) Jaume Martínez Bonafé. Universidad de Valencia F. Javier Merchán Iglesias. Universidad de Sevilla Emilia Moreno Sánchez. Universidad de Huelva. Rosario Ortega Ruiz. Universidad de Córdoba Antonio de Pro Bueno. Universidad de Murcia Fco. de Paula Rodríguez Miranda. Universidad de Huelva

Pedro Sáenz-López Buñuel. Universidad de Huelva Antoni Santisteban Fernández. Universidad Autónoma (Barcelona)

Emilio Solís Ramírez. Catedrático de IES.

$\mathbf{M}^{\mathbf{a}}$ Victoria Sánchez García. Universidad de Sevilla. Magdalena Suárez Ortega. Universidad de Sevilla

\section{Consejo asesor}

Manuel Area Moreira. Universidad de La Laguna

Jaume Carbonell. Director Cuadernos de Pedagogía. Barcelona

César Coll. Universidad de Barcelona

Christopher Day. Universidad de Nothingham. U.K.

Juan Delval. Universidad Nacional de Educación a Distancia

John Elliott. Universidad de East Anglia. Norwich. U.K.

José Gimeno Sacritán. Universidad de Valencia

André Giordan. Universidad de Paris VII y Ginebra

Francisco Imbernón. Universidad de Barcelona

Ángel Pérez Gómez. Universidad de Málaga

Rafael Porlán Ariza. Universidad de Sevilla

Francesco Tonucci. Instituto de Pedagogía del C.N.R. Roma

Jurjo Torres Santomé. Universidad de A Coruña

La revista Investigación en la Escuela desde su origen en 1987 hasta su nº 87 (2015) fue editada por Díada Editora. 